\title{
A Halal and Quality Attributes Driven Animal Products Formal Producing System Based on HQESPNM
}

\author{
Qiang Han* and Wenxing Bao \\ School of Computer Science and Engineering, BeiFang University of Nationalities, Yinchuan \\ Ningxia, P.R. China 750021 \\ nxhanq@gmail.com
}

\begin{abstract}
Usually, halal animal products formal producing system consists of several components that cover major stages including Pre-processing, Processing and Post-processing. In this paper, we present five information systems to implement fundamental functions of formal management, scientific foods management, animal epidemic disease diagnose and prevention, processing standardization and market management, which respectively map to those components mentioned above. As halal animal products formal producing system, there are Halal \& Quality attributes existing in all of the five information systems. Thereby, concentrated and systematic controlling of Halal \& Quality attributes could improve whole quality of the producing system and ensure products is halal. Addressed to the problem of controlling scheme, first, this paper given a Halal \& Quality Elements Extended SPN Model (HQESPNM) in detail. Second, it propose Platform-Independence architecture of the formal producing system based on HQESPNM through infrastructure of database integrate middleware. Finally, this paper given an Electronic-Agriculture Services case through Platform-Specified Software based on SOA to certificate that the model proposed by this paper is feasible for halal animal products system.
\end{abstract}

Keywords: Halal, Quality, Traceability, Petri Nets, HQESPNM.

\section{Introduction}

At the beginning of 80's of last century, based on the Petri Net [1], Molly presented Stochastic Petri Net through associating a stochastic delay time to every transition from ready-to-fire to firing [2]. With the development of science, computation science based on high-performance computing becomes more and more important[5].As the Stochastic Petri Net presented, it was used in many applications of modeling, analysis and efficiency test, such as communication protocols, workflow design etc.

However, Stochastic Petri Net is not suitable to each application aspect completely. For example, to the formal producing and quality certification of Halal Animal, except general elements of normal management are suitable to Stochastic Petri Net for information system modeling and computation, the type of its Halal and Quality elements affection to all of steps in whole management process is through controlling the

${ }^{*}$ Corresponding author.

D. Li, Y. Liu, and Y. Chen (Eds.): CCTA 2010, Part I, IFIP AICT 344, pp. 48-55. 2011.

(C) IFIP International Federation for Information Processing 2011 
process and not by any time-element or stochastic-element. Once the Halal and Quality elements are destroyed in the management process, the products have not any meaning for Moslem people and other people.

Including all the animal product producing process of Pre-processing, Processing and Post-processing, in breeding, propagate, feed, fattening, slaughtering, transportation and market circulating, how to guarantee the products are processed under an consistency environment according to Halal and Quality standard, is the key of information system which service for this aspect.

To solve the problem above, this paper proposed an approach through combination technique [13] of Place expanding Petri nets [6] and Stochastic Petri Net [2], and by the approach, this paper given a Halal \& Quality Elements Extended SPN Model (HQESPNM) in detail. The primary thought of the approach is separate the problem of the aspect into two part, the part about general performance computation can be solved by Stochastic Petri Net, and the other part about Halal \& Quality elements can be solved by Place expanding Petri nets. So that, by this means, through the function of the two Petri net theories, the problem of the aspect can be solved successfully.

In the detailed scheme of the halal animal products formal producing system model based on HQESPNM, this paper mainly given design of the model which separate Halal \& Quality attributes into independent subsystem, and the implementation of its algorithm.

The organization of the remainder of this paper is as follows. Section 2 given the basic concepts and notations, and system model is given in section 3. Section 4 discussed how to use the system to solve the problem through an example. Finally, section 5 summarized the main results and points out the future work. The word "iff" means "if and only if" in this paper.

\section{Basic Concepts and Notations}

The concepts and notations of Petri nets are derived from some documents [7-12].

\subsection{Stochastic Petri Net}

Definition 1. A Petri net is a four-tuple $\sum=\left(P, T, F ; M_{0}\right)$ such that:

(1) $\mathrm{P}$ is a finite set of places, and $\mathrm{T}$ is a finite set of transitions, and $P \bigcap T=\phi, P \bigcup T \neq \phi$;

(2) $F \subseteq(P \times T) \cup(T \times P)$ is a set of arcs;

(3) $M_{0}: P \rightarrow\{0,1,2, \ldots\}$ is the initial marking. Generally, $\forall x \in P \bigcup T$, the preset of $\mathrm{x}$ is $x=\{y \mid y \in P \bigcup T$ and $(\mathrm{y}, \mathrm{x}) \in F\}$, and the post-set of $\mathrm{x}$ is $x=\{y \mid y \in P \bigcup T$ and $(\mathrm{x}, \mathrm{y}) \in F\}$ and $x \bigcup x$ is the spanning-set of $\mathrm{x}$. If $P_{1}=P, T_{1}=T$ and $\mathrm{F}_{1} \in\left(\left(P_{1} \times T_{1}\right) \cup\left(T_{1} \times P_{1}\right)\right) \cup F, \sum_{1}=\left(P_{1}, T_{1}, F_{1}\right)$ is called the spanning subnet of $\mathrm{P} 1$. 
Definition 2. Let $\mathrm{M}(\mathrm{p})$ be the number of tokens in place $\mathrm{p}$. For $t \in T$,

(1) $\mathrm{t}$ is enable under the marking $\mathrm{M}$, denoted by $M[t>$,iff $\forall p \in t: M(p) \geq 1$; ;

(2) If $t$ is enable under the marking $M$, then $t$ can be fired. The marking

$M^{\prime}$ is obtained from $\mathrm{M}$ by firing $\mathrm{t}$, denoted by $M\left[t>M^{\prime}\right.$ :

$$
M^{\prime}(p)=\left\{\begin{array}{c}
M(p)-1,(p, t) \in F \wedge(t, p) \notin F \\
M(p)+1,(p, t) \notin F \wedge(t, p) \in F \\
M(p), \text { otherwise }
\end{array}\right.
$$

Thus, $\mathbf{M}^{\prime}$ is reachable from $\mathbf{M}$. The set of reachable markings from $\mathbf{M}$ is denoted as $\mathrm{R}(\mathrm{M})$.

The properties derived from execution of the Petri net are called dynamic properties or behavioral properties.

A Petri net $\sum=\left(P, T, F ; M_{0}\right)$ is called safe

iff $\forall M \in R\left(M_{0}\right), \forall p \in P, M(p) \leq 1$ is satisfied.

Definition 3. For a Petri net $\sum=\left(P, T, F ; M_{0}\right)$,

$M \in R\left(M_{0}\right)$, if $M \in R\left(M^{\prime}\right)$ for $\forall M^{\prime} \in R(M)$, then $\mathrm{M}$ is called a home state. $\sum$ is a reversible net system if $M_{0}$ is a home state.

Definition 4. $\sum=\left(P, T, F ; M_{0}\right)$ is a Petri net. $\sum$ is said to be:

(1) weakly live iff $\forall M \in R\left(M_{0}\right), \exists t \in T$ such that $M[t>$.

(2) live iff $\forall M \in R\left(M_{0}\right), \exists t \in T, \exists \sigma \in T^{*}$ such that $M\left[\sigma>M^{\prime}[t>\right.$.

Definition 5. $\sum=\left(P, T, F ; M_{0}, K, W\right)$ is a Place/Transition Net, where:

(1) $\left(P, T, F ; M_{0}\right)$ is a Petri net.

(2) $K: S \rightarrow N^{+} \bigcup\{\infty\}$ is Place Capacity function.

(3) $W: F \rightarrow N^{+}$is Arc Priority function.

(4) $\forall p \in P: M_{0}(p) \leq K(p)$.

Definition 6. $\sum=\left(P, T, F ; M_{0}, K, W, \lambda\right)$ is a continuous-timed Stochastic Petri Nets, where:

(1) $\left(P, T, F ; M_{0}, K, W\right)$ is a Place/Transition Net.

(2) $\lambda=\left(\lambda_{1}, \lambda_{2}, \lambda_{3}, \cdots, \lambda_{m}\right)$ is set of transition average fired rate. $\lambda_{i}$ is transition average fired rate of $t_{i} \in T$, which represent the fired times of $t_{i}$ in an unit time.

\subsection{Place Expanding Petri Net (PePN)}

Based on the introduction of Petri Nets and Stochastic Petri Nets above, we can calculate performance data through modeling system. However, in the actual application of 
it, because of the dynamic change properties of modeling objects, the capacity of description for them is limited. So some researchers presented Place expanding Petri Net Models, example Place expanding Petri Net[6].Its definition is:

Definition 7. $\sum=\left(P, T, F ; M_{0}\right)$ is a Place expanding Petri Net(PePN), where:

(1) $\mathrm{S}=\left\{\right.$ sls is Place expanding Petri Net or $s \subseteq S^{\prime}, S^{\prime}$ is place sets of $\sum$.

(2) $\forall(x, y) \in F: x \in S \wedge y \in T \Rightarrow \exists z \in S,(y, z) \in F$.

(3) $\forall(x, y) \in F: x \in T \wedge y \in S \Rightarrow \exists z \in T,(z, x) \in F$.

\subsection{Halal and Quality Elements Extended SPN Model (HQESPNM)}

Based on the Stochastic Petri Nets and Place expanding Petri Net, this paper presented a Halal\&Quality Elements Extended SPN Model (HQESPNM).

Definition 8. $\sum=(N h, N q, N p, F)$ is a HQESPNM, where:

(1) $N h=\left(P_{N h}, T_{N h}, F_{N h} ; M_{0}^{N h}\right)$ is a Petri Nets.

(2) $N q=\left(P_{N q}, T_{N q}, F_{N q} ; M_{0}^{N q}\right)$ is a Petri Nets.

(3) $N p=\left(P_{N p}, T_{N p}, F_{N p} ; M_{0}^{N p}, K_{N p}, W_{N p}, \lambda_{N p}\right)$ is a Stochastic Petri Nets.

(4) $F=\left\{<p, t>\cup<t, p>\mid p \in\left(P_{N h} \cup P_{N q}\right), t \in T_{N p}\right\}<p, t>$ means a Halal or Quality data flow/transition from $N h$ or $N q$ to $N p$; on the contrary, $<t, p>$ means a Halal or Quality data flow/transition from $N p$ to $N h$ or $N q$.

For the application in the Introduction of this paper, $N h$ represent abstract of Halal-element relation, $N q$ represent abstract of Quality-element relation, $N p$ represent abstract of Animal Products Producing, $F$ represent abstract of controlling from $N h$ and $N q$ to $N p$ and reflection from $N p$ to $N h$ and $N q$.

Based on the HQESPNM, we can find that the correctly systematically running of $N p$ has to be controlled under $N h$ and $N q$ through $F$. Then this paper given the system architecture and algorithm to implement HQESPNM as follow.

\section{System Model}

In this section, we gave a Meta-model of Compute-Independence (CIM) for HQESPNM firstly. For the system design, according to the CIM, a Model of Platform-Independence (PIM) for HQESPNM was given through approach of OOA\&OOD [3][4]. In the PIM, we illustrated the relation between $N h$ and $N p$ as well as the relation between $N q$ and $N p$. 


\subsection{System Architecture}

The basic components of halal products formal producing system includes:

(1) Formal Management Information System (FMIS).

(2) Processing Standardization Information System (PSIS).

(3) Market Management Information System (MMIS).

(4) Scientific Food Management Information System.

(5) Animal Epidemic Diagnose Information System.

(6) Quality Traceability Information System (QTIS).

(7) Halal Traceability Information System (HTIS)

(8) Public Data Traceability Information System.

(9) Traceability Data Bus based on Data Integrity Middleware.

Among the components above:

(1),(2) and (3) consists of the $N p$ in HQESPNM, which is the major body of halal products formal producing system, representing Pre-processing, Processing and Postprocessing stage respectively.

(4) and (5) assist the normal running of $\mathrm{Np}$. (4) is a non-independent component of the Formal Management Information System, on the contrary, (5) is an independent component based on artificial intelligence, which can be loaded or unloaded freely.

(6) and (7) consists of the $N q$ and $N h$ respectively in HQESPNM, which are the kernel of halal products formal producing system.

(8) is a trusted third-party component, which provide common qualification data to the $N q$ via exchange interface of (9).

(9) is Traceability Data Bus based on Data Integrity Middleware, connecting the components mentioned above as a whole system, assurance the $N p$ could be operated under the controlling of $N q$.

CIM of system architecture can be described as Fig.1.

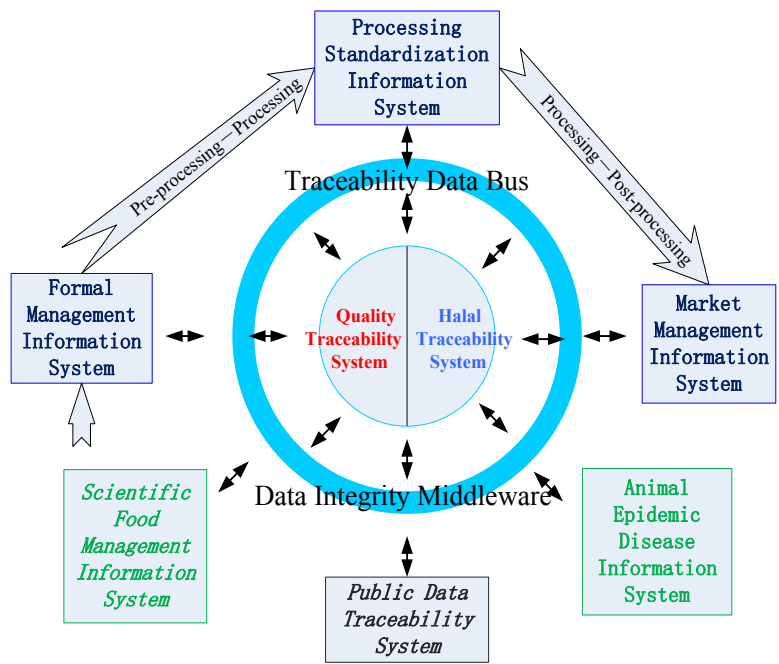

Fig. 1. CIM of System Architecture 


\subsection{Design of $F$ in HQESPNM}

According to the CIM of System Architecture above, next, through the theory of Stochastic Petri Nets and UML, we can map components and complex connectors into package, function specifications into interfaces, entry points into abstract class, and inner specification of component into comments respectively. Then, according to $F$ in the definition 8, we can give the kernel PIM of System Architecture concentrated in relation between $N h, N q$ and $N p$ as Fig.2, which reflects the $F$ in HQESPNM.

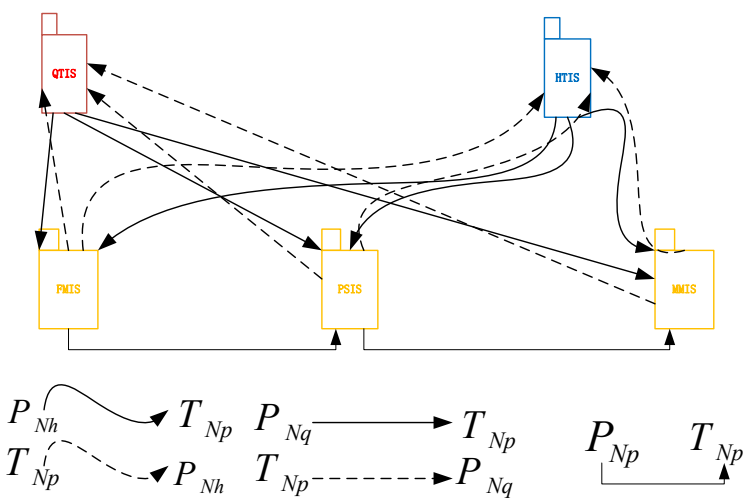

Fig. 2. $F$ in HQESPNM

\section{Overview of HQESPNM by Example}

Based on the research above, we developed a set of software prototype named as Hqespnm1.0 to certificate the correctness of the HQESPNM. In this section, we presented an example to introduce the set of software prototype as Fig.3.

Hqespnm1.0 consists of Halal Traceability Information System (HTIS) and Quality Traceability Information System (QTIS), which show as upper part and Lower half of Fig.3 respectively.

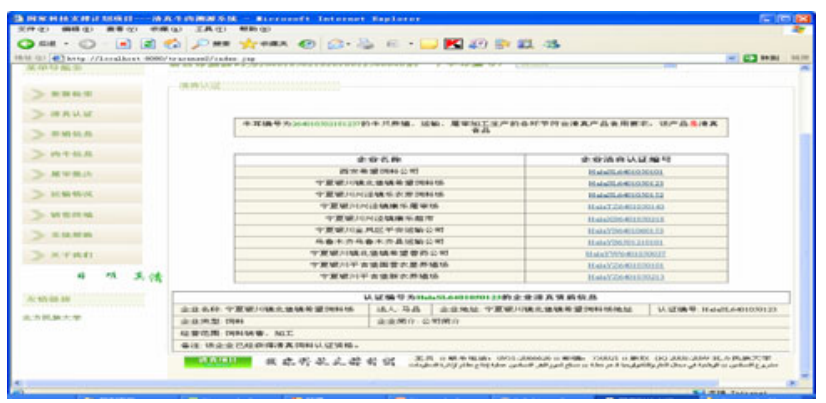

Fig. 3. Hqespnm1.0 based on HQESPNM 


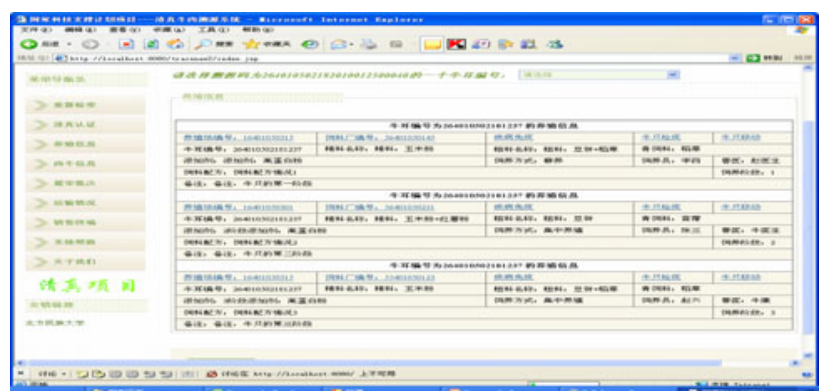

Fig. 3. (continued)

\section{Conclusion and Future Working}

Address to Halal \& Quality attributes assurance problem of formal producing system, the basic concept and notation of HQESPNM is given firstly, and the software model including architecture and design is presented secondly. Based on the model, this paper introduced an application example in electronic agriculture domain covering whole producing process including Pre-producing, Producing, Post-producing finally.

Results indicated that the HQESPNM separate the normal producing elements and Halal \& Quality controlling elements into Petri Nets and Stochastic Petri Nets through Place expanding Petri Net [6] successfully.

In future, the formal work of HQESPNM should be implemented to certificate its correctness.

\section{Acknowledgement}

This paper is supported by National Key Technology R\&D Program of China under Grant No.2007BAD33B03, Natural Science Foundation of NingXia Province under Grant No.NZ0955 and the Colleges Oriented Scientific Research Fund of NingXia Provincial Education Department of China under Grant No.2008JY009. Additionally, we should thank for the software prototype development work distributed by our colleagues and master candidate students: Ding HongSheng, Yang YongSheng, Shi Liang and Liu Yang.

\section{References}

1. Petri, C.A.: Kommunkation mit automaten. Schriften des IIM, vol. 3. Institut fur Lnstrum Entelle Mathematik, Bonn (1962)

2. Molly, M.K.: Discrete time stochastic Petri nets. IEEE Trans. Software Eng. SE-11(4), 417-423 (1985)

3. Shao, W.-z., Yang, F.-q.: Object-Oriented System Anysis. Publishing House of Tsinghua University (2006) 
4. Shao, W.-z., Yang, F.-q.: Object-Oriented System Design. Publishing House of Tsinghua University (2007)

5. Cui, H.-q., Wu, Z.-h.: MPI Programs' Petri Net Model and Its Dynamic Properties. Journal of System Simulation 18(9), 2455-2460 (2006)

6. Qi, F.-m., Yu, B., Shi, L.-j., Mou, L.-k.: A Modeling Method of Software Project Management Based on Petri Nets. Journal of System Simulation 19(suppl. 1), 75-78 (2007)

7. Peterson, J.L.: The Theory of Petri Net and System Simulation. Wu Zhehui (Trans). Publishing House of China University of Mining Technology, Xuzhou (1989)

8. Murata, T.: Petri Nets: Properties,Analysis and Applications. Proceedings of the IEEE(S0018-9219) 77(4), 541-580 (1989)

9. Yuan, C.: The Principles of Petrinet. Publishing House of Electronics Industry, Beijing (2005)

10. Lin, C.: Stochastic Petri Nets and System Performance Evaluation. Publishing House of Tingshua University, Beijing (2005)

11. Zhan, H., Gu, J.,: Study of the Normal Generalized Stochastic Petri nets and its Application in Testing System. In: IEEE Instrumentation and Measurement Technology Conference Proceedings, pp. 1123-1128 (2006)

12. Renato Vazquez, C., Recalde, L., Silva, M.: Stochastic Continuous-State Approximation of Markovian Petri Net Systems. In: Proceedings of the 47th IEEE Conference on Decision and Control, pp. 901-906 (2008)

13. Han, Q., Ding, J., Bao, W.: IEEE Proceedings of the 2009 International Conference on Computer and Computing Technology Applications in Agriculture (2009) 